\title{
Reflets
}

Revue d'intervention sociale et communautaire

\section{Le mieux-être des travailleurs sociaux au travail}

\section{Claude Larivière}

Volume 19, numéro 2, automne 2013

La souffrance psychique et morale au travail : enjeux pour les professionnels du secteur de la santé et des services sociaux

URI : https://id.erudit.org/iderudit/1021180ar

DOI : https://doi.org/10.7202/1021180ar

Aller au sommaire du numéro

Éditeur(s)

Reflets, Revue d'intervention sociale et communautaire

ISSN

1203-4576 (imprimé)

1712-8498 (numérique)

Découvrir la revue

Citer cet article

Larivière, C. (2013). Le mieux-être des travailleurs sociaux au travail. Reflets, 19(2), 72-84. https://doi.org/10.7202/1021180ar

\section{Résumé de l'article}

Les travailleurs sociaux sont sensibles au climat de travail dans lequel ils effectuent leurs interventions au sein des organismes. Au Québec, certains organismes réussissent à les soutenir alors que d'autres génèrent de l'insatisfaction. Cet article explore les facteurs qui influencent ces perceptions et fait état d'une démarche de l'Ordre des travailleurs sociaux et des thérapeutes conjugaux et familiaux du Québec.
Tous droits réservés (C) Reflets, Revue d'intervention sociale et communautaire, 2013
Cecument est protégé par la loi sur le droit d'auteur. L'utilisation des services d'Érudit (y compris la reproduction) est assujettie à sa politique d'utilisation que vous pouvez consulter en ligne.

https://apropos.erudit.org/fr/usagers/politique-dutilisation/ 


\section{Le mieux-être des travailleurs sociaux au travail}

\section{Claude Larivière}

Professeur associé

Directeur des programmes facultaires d'administration sociale (FAS), Université de Montréal

\section{Résumé}

\section{Abstract}

Les travailleurs sociaux sont sensibles au climat de travail dans lequel ils effectuent leurs interventions au sein des organismes. Au Québec, certains organismes réussissent à les soutenir alors que d'autres génèrent de l'insatisfaction. Cet article explore les facteurs qui influencent ces perceptions et fait état d'une démarche de l'Ordre des travailleurs sociaux et des thérapeutes conjugaux et familiaux du Québec.

Mots clés : Climat de travail, soutien professionnel

The management of social workers is a delicate subject as many of them Love their job with clients but " hate " the organization who employ them because bad employment conditions, lack of Professional support, etc. This article is about factors related to the construction of these perceptions.

Key words : Work climate, Professional support

Les médias rapportent assez fréquemment des situations où le climat de travail d'un établissement public est décrié par son 
personnel ou par le syndicat qui le représente, tel l'impact appréhendé de projets d'optimisation et de réorganisation du travail. Loin d'être des cas isolés, ces situations témoignent de l'existence de difficultés dans la gestion des ressources humaines et plus particulièrement chez les professionnels des services de santé et des services sociaux. Souvent, les intervenants sociaux jouent un rôle de premier plan dans ces sorties sur la place publique et cela s'explique certainement par une sensibilité plus grande en raison du fait que le travail social est au cœur des tensions personnelles, familiales, interpersonnelles et communautaires que vivent les gens.

Ce n'est pas d'hier que des auteurs soulignent l'impact du stress professionnel (Aubert et Pagès, 1989; Boyer, et collab., 1995; Béland, 2009) et le risque de burnout des travailleurs sociaux (Pezet,Villatte et Logeay, 1993) vu comme une occasion de se mobiliser pour passer de l'usure à une reconstruction de l'identité professionnelle. Il existe même un guide à l'intention des intervenants sociaux pour les aider à faire échec au burnout (Arcand et Brissette, 1998). Quant au harcèlement moral que peuvent exercer certains employeurs ou parfois des collègues de travail, Hirigoyen (2001) a distingué "le vrai du faux ", comme elle l'écrit, de façon à nous sensibiliser à ce phénomène de société.

"Toutes ces organisations s'avèrent soumises à des contraintes assez semblables : pénurie de ressources, maind'œuvre vieillissante, nécessité de s'adapter à de nouveaux besoins, contraintes budgétaires, listes d'attente, informatisation accrue et développement de réseaux intégrés de services. »
Parallèlement pourtant, d'autres organisations publiques et communautaires parviennent à relever le défi de l'implication de leur personnel et réussissent à s'assurer que leurs équipes de travail développent un haut niveau de cohésion et de complémentarité. Toutes ces organisations s'avèrent soumises à des contraintes assez semblables : pénurie de ressources, maind'œuvre vieillissante, nécessité de s'adapter à de nouveaux besoins, contraintes budgétaires, listes d'attente, informatisation accrue et développement de réseaux intégrés de services.

Comment, du point de vue du personnel qui y travaille quotidiennement, expliquer de telles variations? Et ensuite, quelles bonnes pratiques pouvons-nous encourager et développer? Tel est le propos de cet article qui, bien que basé essentiellement sur des données tirées de recherches québécoises, s'inspire largement d'écrits internationaux. Il va aussi dans le sens d'une 
étude canadienne en cours de réalisation avec l'aide de plusieurs associations provinciales et présentée en juin 2013 au Congrès des sciences sociales et humanités (Bejan, 2012). Il faut toutefois noter qu'il s'agit ici essentiellement d'un bilan critique et non pas d'une nouvelle recherche.

\section{La situation dans les établissements publics québécois}

Le réseau d'établissements publics québécois offrant des soins de santé et des services sociaux emploie près de $10 \%$ de la maind'œuvre du Québec, soit plus de 220000 personnes. Notons que seulement 100000 d'entre elles travaillent à temps complet. Cette main-d'œuvre est également féminine à $75 \%$ et mobilise $30 \%$ du budget des services de l'État. Or, année après année, un pourcentage important des heures rémunérées n'est pas travaillé, ce qui traduit certainement un malaise sérieux :

Les établissements du réseau de la santé et des services sociaux ont connu depuis 1993-1994 une augmentation des absences en assurancesalaire de l'ordre de $24 \%$. [...] Environ 10500 employés en équivalant à temps complet sont absents annuellement en fonction de l'un ou [de] l'autre des régimes, ce qui représente 7,5\% des effectifs à temps complet du réseau; les coûts directs des régimes d'indemnisation se situent à plus de 330 millions de dollars annuellement. (Ministère, 2001, p. i)

L'adaptation aux besoins perçus de la population a souvent pris la forme de changements de structures (fusion d'établissements, restructurations complètes en 2004) de vocations et de transferts d'effectifs. Or, ces changements majeurs provoquent des chocs culturels qui persistent souvent pendant plusieurs années et se traduisent difficilement à court terme en gains d'efficacité en ce qui a trait à l'intégration accrue des services, à la complémentarité des expertises et à la coordination facilitée (Collerette, 2008).

Une recherche américaine (Glisson et Hemmelgarn, 1998) a mis en évidence l'importance du climat organisationnel pour 
"Pour une majorité de répondants, le climat de travail est décrit comme difficile, bien qu'il existe également des milieux stimulants. Cela nous permet de poser une question fondamentale : selon quels facteurs des employés peuventils estimer que le climat de travail dans leur organisation est favorable ou non? " faciliter la collaboration interorganisationnelle et assurer de la complémentarité dans les services aux jeunes au Tennessee. Les auteurs expliquent que dans un milieu de travail où les gestionnaires font confiance aux professionnels, ceux-ci sont davantage à l'aise de prendre des initiatives et utilisent leur autonomie à bon escient.

La situation au Québec n'est pas très différente selon une recherche menée dans les services aux jeunes en difficulté (Larivière, 2001). Pour une majorité de répondants, le climat de travail est décrit comme difficile, bien qu'il existe également des milieux stimulants. Cela nous permet de poser une question fondamentale : selon quels facteurs des employés peuvent-ils estimer que le climat de travail dans leur organisation est favorable ou non? L'analyse de ces facteurs permet ensuite de dresser une liste des bonnes et mauvaises pratiques de gestion des ressources humaines dans un établissement public où les professionnels constituent l'ossature autour de laquelle gravitent les services à la clientèle. Nous aborderons successivement deux types de facteurs : 1. intrapersonnels et liés aux valeurs; 2 . intra-organisationnels et reliés au mode de gestion de l'organisme employeur.

La première dimension, celle des facteurs intrapersonnels s'explique par la motivation intrinsèque qui conduit une majorité de personnes à exercer une profession en vue de se réaliser en raison d'une valorisation de la nature particulière de leur tâche (les services à rendre, l'expertise à utiliser, le soin à donner) dans un contexte qu'elles souhaitent respectueux de leur autonomie professionnelle (Glisson et Hemmelgarn, 1998).

Nous savons que les travailleurs sociaux manifestent souvent de l'empathie pour les personnes auprès desquelles ils interviennent, que plusieurs d'entre eux s'engagent autour de causes mobilisatrices liées à la victimisation d'une partie de la clientèle de leurs services, que l'empowerment signifie aussi aider les usagers à se solidariser et à défendre leurs droits. Logiquement et professionnellement, les usagers constituent la raison d'être des travailleurs sociaux, sans oublier les enjeux plus collectifs et communautaires en lien avec des perspectives de changement et d'amélioration des politiques sociales. 
Tableau 1 : Les facteurs intrapersonnels qui contribuent à construire la perception du climat de travail (synthèse tirée de Larivière, 2001, p. 112-115)

\begin{tabular}{|c|c|c|}
\hline & Mauvais climat & Bon climat \\
\hline $\begin{array}{l}\text { Les valeurs } \\
\text { personnelles }\end{array}$ & $\begin{array}{l}\text { - la clientèle n'est pas véritablement } \\
\text { au centre des préoccupations } \\
\text { organisationnelles; } \\
\text { - impossibilité de pratiquer selon } \\
\text { les règles de l'art, sentiment } \\
\text { d'impuissance et pas d'espoir } \\
\text { d'amélioration. }\end{array}$ & $\begin{array}{l}\text { - préoccupation partagée du client; } \\
\text { - possibilité de se réaliser } \\
\text { professionnellement; } \\
\text { - personnel disposé à apprendre et } \\
\text { ouvert au changement. }\end{array}$ \\
\hline $\begin{array}{l}\text { La tâche } \\
\text { a accomplir }\end{array}$ & $\begin{array}{l}\text { - niveau constant de stress élevé, forte } \\
\text { pression intense (liste d'attente); } \\
\text { - compétences mal ou sous-utilisées, } \\
\text { isolement clinique; } \\
\text { - précarité d'emploi et obligation de se } \\
\text { réadapter à chaque changement de } \\
\text { tâche. }\end{array}$ & $\begin{array}{l}\text { - valorisation du travail clinique, travail } \\
\text { reconnu et apprécié, diversité des } \\
\text { défis; } \\
\text { - le climat de travail est stimulant et le } \\
\text { soutien permet de réaliser la tâche } \\
\text { malgré le stress; } \\
\text { - sentiment que l'organisation fait du } \\
\text { très bon travail. }\end{array}$ \\
\hline $\begin{array}{l}\text { L'autonomie } \\
\text { professionnelle }\end{array}$ & $\begin{array}{l}\text { - absence d'autonomie décisionnelle, } \\
\text { grande imputabilité et peu de pouvoir. }\end{array}$ & $\begin{array}{l}\text { - respect de l'autonomie } \\
\text { professionnelle à l'intérieur des } \\
\text { balises et des critères connus. }\end{array}$ \\
\hline
\end{tabular}

Malheureusement, le personnel constate souvent que les enjeux budgétaires déterminent souvent les décisions organisationnelles, que la pression de la demande sur les services fait en sorte que la quantité menace la qualité dans un contexte où l'autonomie décisionnelle peut devenir factice. Les projets d'optimisation qui peuvent améliorer l'organisation parfois routinière du travail peuvent aussi, selon la façon dont ils sont amenés par les gestionnaires, paraître comme des menaces à l'autonomie dans l'organisation de son travail quotidien.

Dans un mauvais climat, le niveau de stress peut être très élevé et affecter la capacité de certains intervenants plus fragiles de faire face aux exigences de leur employeur, les mener à commettre des erreurs ou encore à devenir malade. Ici encore, les éléments organisationnels viennent affecter les individus qui y travaillent. 
"Malheureusement, le personnel constate souvent que les enjeux budgétaires déterminent souvent les décisions organisationnelles, que la pression de la demande sur les services fait en sorte que la quantité menace la qualité dans un contexte où l'autonomie décisionnelle peut devenir factice. "

"C'est toutefois autour des facteurs reliés à la gestion que se cristallise habituellement l'éloignement entre les valeurs personnelles, professionnelles et le mode de fonctionnement de l'environnement de travail."
Le travail social d'accompagnement de la souffrance sociale n'est jamais aisé et laisse des traces dans la conscience de ceux qui le pratiquent. Le problème est suffisamment important pour que la revue Intervention lui consacre en 2002 un numéro dans lequel Couturier et Legault (p. 57-63) soulignent l'importance de renforcer l'identité professionnelle des travailleurs sociaux pour faire justement face à la complexité de la tâche. Des professionnels affirmés, maîtrisant adéquatement modèles et outils d'intervention, auront moins tendance à se sentir menacés et stressés par les difficultés normales rencontrées dans leur pratique.

Cet enjeu n'est évidemment pas nouveau puisque Hopkins (1983) abordait la question de la relation entre les professionnels de la fonction publique et cet environnement bureaucratique à partir des travaux de la psychologie organisationnelle de l'après-Seconde Guerre mondiale caractérisée par une approche humaniste.

C'est toutefois autour des facteurs reliés à la gestion que se cristallise habituellement l'éloignement entre les valeurs personnelles, professionnelles et le mode de fonctionnement de l'environnement de travail.

La deuxième dimension aborde justement un ensemble de facteurs internes à l'organisation soit la gestion - ou la nongestion - du changement, le style de gestion et le leadership assumé par les cadres, les tensions provoquées par l'inadéquation des ressources disponibles et l'ensemble des processus mis en place pour faciliter l'efficacité organisationnelle.

Ces aspects sollicitent grandement les habiletés des gestionnaires. Le contexte historique du secteur public où le personnel adhère obligatoirement à des organisations de type syndical a trop souvent contribué à transformer la gestion des ressources humaines en une gestion des relations de travail. L'utilisation optimale des ressources nécessite souvent une réorganisation du travail (création d'équipes par programme-clientèle, encouragement à l'interdisciplinarité, gestion de cas et imputabilité accrue, participation à des équipes interorganisationnelles dans le cas de services complexes). Ce sont là des changements que bien peu de cadres se sentent aptes à piloter et des défis de gestion des pratiques qui demandent du 
soutien de gestionnaires trop souvent non disponibles. Cela ressort plus particulièrement du tableau suivant :

Tableau 2 : Les facteurs intra-organisationnels qui contribuent à construire la perception du climat de travail (synthèse tirée de Larivière, 2001, p. 120-126)

\begin{tabular}{|c|c|c|}
\hline & Mauvais climat & Bon climat \\
\hline $\begin{array}{l}\text { La gestion du } \\
\text { changement }\end{array}$ & $\begin{array}{l}\text { - instabilité, changements } \\
\text { technologiques sans préparation; } \\
\text { - restructuration lente et pénible, } \\
\text { pas de culture organisationnelle } \\
\text { partagée, adaptation à de } \\
\text { multiples changements } \\
\text { simultanés. }\end{array}$ & $\begin{array}{l}\text { - les décisions liées à la } \\
\text { transformation organisationnelle } \\
\text { se prennent en concertation; } \\
\text { - organisation capable de se } \\
\text { remettre en question, place pour } \\
\text { l'innovation. }\end{array}$ \\
\hline Le style de gestion & $\begin{array}{l}\text { - absence de vision quant aux } \\
\text { services (l'administratif domine } \\
\text { le clinique), fonctionnement } \\
\text { technocratique, contrôle excessif, } \\
\text { manque de cohésion au sein de } \\
\text { l'équipe de gestion; } \\
\text { - absence de vision quant aux } \\
\text { services (l'administratif domine } \\
\text { le clinique), fonctionnement } \\
\text { technocratique, contrôle excessif, } \\
\text { manque de cohésion au sein de } \\
\text { l'équipe de gestion; }\end{array}$ & $\begin{array}{l}\text { - contacts cordiaux entre } \\
\text { gestionnaires et intervenants, } \\
\text { grande confiance dans la } \\
\text { compétence des professionnels, } \\
\text { diverses formes de participation; }\end{array}$ \\
\hline $\begin{array}{l}\text { Le style de gestion } \\
\text { (suite) }\end{array}$ & $\begin{array}{l}\text { - réaménagement des tâches } \\
\text { sans implication du personnel, } \\
\text { pseudoconsultation ou absence de } \\
\text { consultation, méfiance réciproque } \\
\text { entre gestionnaires et employés; } \\
\text { - valorisation de la performance } \\
\text { (quantité) plutôt que de la qualité, } \\
\text { pas de connaissance du travail } \\
\text { accompli, pas de volonté de régler } \\
\text { les problèmes identifiés. }\end{array}$ & $\begin{array}{l}\text { - bonne cohésion de l'équipe de } \\
\text { gestion, gestionnaires orientés } \\
\text { vers les services à la clientèle; } \\
\text { - direction transparente, projet } \\
\text { organisationnel construit avec la } \\
\text { participation du personnel. }\end{array}$ \\
\hline
\end{tabular}


Tableau 2 - (suite) : Les facteurs intra-organisationnels qui contribuent à construire la perception du climat de travail (synthèse tirée de Larivière, 2001, p. 120-126)

\begin{tabular}{|c|c|c|}
\hline & Mauvais climat & Bon climat \\
\hline $\begin{array}{l}\text { Les ressources } \\
\text { diponibles }\end{array}$ & $\begin{array}{l}\text { - prétendre offrir des services } \\
\text { (quantité, qualité) avec des } \\
\text { ressources insuffisantes, locaux } \\
\text { peu adéquats. }\end{array}$ & $\begin{array}{l}\text { - reconnaissance de la pénurie de } \\
\text { ressources et effort pour pallier } \\
\text { ce manque selon les priorités } \\
\text { déterminées en consultation avec } \\
\text { le personnel. }\end{array}$ \\
\hline $\begin{array}{l}\text { Les processus mis en } \\
\text { place }\end{array}$ & $\begin{array}{l}\text { - absence complète de soutien } \\
\text { (organisationnel, clinique) et } \\
\text { de supervision (insatisfaction } \\
\text { à l'égard de celle-ci), pas de } \\
\text { formation continue, essoufflement } \\
\text { du personnel, absence de } \\
\text { reconnaissance, croissance du } \\
\text { pourcentage d'occasionnels, perte } \\
\text { de l'expertise (départs); }\end{array}$ & $\begin{array}{l}\text { - recherche constante de } \\
\text { solutions pour le soutien du } \\
\text { personnel, développement de } \\
\text { l'interdisciplinarité, supervision } \\
\text { clinique, reconnaissance } \\
\text { des réussites, intégration et } \\
\text { soutien (formation, supervision) } \\
\text { des occasionnels, plan de } \\
\text { développement des ressources } \\
\text { humaines actualisé; }\end{array}$ \\
\hline $\begin{array}{l}\text { Les processus mis en } \\
\text { place (suite) }\end{array}$ & $\begin{array}{l}\text { - absence ou mauvaise circulation } \\
\text { de l'information, peu de continuité } \\
\text { dans les services, pas d'évaluation } \\
\text { de l'impact des décisions, } \\
\text { insatisfaction de la clientèle, listes } \\
\text { d'attente de plusieurs mois; } \\
\text { - tutelle ou inspection, faible } \\
\text { crédibilité de la direction (absence } \\
\text { de leadership), conflits jamais } \\
\text { résolus, relations tendues } \\
\text { avec les syndicats, instabilité } \\
\text { organisationnelle prolongée. }\end{array}$ & $\begin{array}{l}\text { - respect de l'essoufflement du } \\
\text { personnel et reconnaissance du } \\
\text { stress lié au travail, peu de congés } \\
\text { de maladie, climat détendu, } \\
\text { humour, activités sociales } \\
\text { partagées; } \\
\text { - stabilité des intervenants et } \\
\text { des gestionnaires, absence de } \\
\text { liste d'attente, forte cohésion } \\
\text { interne et bonne identification } \\
\text { à l'organisation, amélioration } \\
\text { continue des services. }\end{array}$ \\
\hline
\end{tabular}

L'Ordre des travailleurs sociaux et des thérapeutes conjugaux et familiaux du Québec (OTSTCFQ) (2010) s'est préoccupé de ces aspects à l'occasion de la restructuration majeure qu'a connue le réseau public québécois à partir de 2004. Le diagnostic qui s'en dégage est assez clair : 
- Tout d'abord, un constat voulant que la gestion du changement dans le réseau de la santé et des services sociaux constitue une faiblesse des organisations et d'un grand nombre de gestionnaires, ce qu'avait aussi signalé Collerette (2008). Des gestionnaires, mal préparés, peinaient à assumer le leadership nécessaire pour piloter leur organisation dans un climat d'incertitude et de multiples changements.

- Si certains résultats positifs peuvent être signalés, c'est malheureusement l'ampleur des difficultés (majorité de travailleurs sociaux réaffectés à de nouvelles tâches, charge de cas accrue, perte d'expertise, manque de soutien et d'encadrement, etc.) partagées par les intervenants et corroborées par des gestionnaires sociaux qui méritent avant tout notre attention. Plusieurs de leurs constats sont corroborés par le rapport sur l'Évaluation de l'implantation des réseaux locaux de services de santé et de services sociaux du Ministère (2010) sur l'impact de la restructuration.

- Les cadres vivent eux-mêmes beaucoup d'isolement et se sentent débordés par la tâche, dans des organisations dont le style de gestion est de plus en plus de type traditionnel et de grande taille où les aspects relationnels et humains perdent de leur importance. Même l'association des employeurs, l'Association québécoise des services de santé et des services sociaux (AQESSS) (2010) souligne le risque que la gestion des ressources humaines soit négligée.

Ces différents constats rejoignent aussi d'autres études entreprises au cours des dernières années, notamment par Bourque (2009) et une équipe de chercheurs (Lachapelle, et collab., 2011) intéressés à mesurer l'impact de la transformation des pratiques psychosociales dans le programme Perte d'autonomie liée au vieillissement (PALV).

\section{Discussion autour de ces enjeux}

Si les organisations peuvent souligner à juste titre que les ressources dont elles disposent ne leur permettent pas d'équilibrer l'offre de services avec la demande exprimée, elles n'ont guère 
"Si les organisations peuvent souligner à juste titre que les ressources dont elles disposent ne leur permettent pas d'équilibrer l'offre de services avec la demande exprimée, elles n'ont guère d'excuses pour ne pas se doter d'un projet mobilisateur, prévenir l'essoufflement de leur personnel et concrétiser le Plan de développement des...» d'excuses pour ne pas se doter d'un projet mobilisateur, prévenir l'essoufflement de leur personnel et concrétiser le Plan de développement des ressources humaines (PDRH) prévu par la Loi québécoise sur les services de santé et les services sociaux depuis 1991.

Les facteurs organisationnels, comme le style de gestion, une moindre préoccupation pour les ressources humaines et le soutien organisationnel, empêchent alors les gestionnaires de créer un meilleur climat de travail. Nous devons reconnaitre que ces facteurs ne reçoivent pas suffisamment d'attention sauf lorsque des efforts sont nécessaires pour l'obtention ou le renouvellement d'un agrément (Conseil canadien d'agrément des établissements de santé, Conseil québécois d'agrément).

La littérature (Brunet et Savoie, 1999; Larivière, 2001) indique que les gestionnaires ne constituent pas un groupe uniforme et qu'une partie significative d'entre eux manifeste une sensibilité à l'égard des besoins et du vécu du personnel qu'ils encadrent, malgré les contraintes organisationnelles. Malheureusement, si cette préoccupation n'est pas partagée par leurs supérieurs, il leur est difficile de gérer différemment au quotidien.

Évidemment, un construit comme le climat de travail (Laflamme, 1994) ne se travaille pas aisément et plusieurs aspects doivent être pris en considération au moment de l'évaluer (Brunet et Savoie, 1999). Le contexte des vingt dernières années, marquées par différents courants dont certains sont directement issus du secteur privé, a forcé le passage progressif de l'idéal moderne d'accessibilité universelle aux services vers une gestion postmoderne où les ressources doivent être utilisées au maximum : amélioration continue, affirmation des droits des usagers, déficit zéro, imputabilité et gouvernance. Le danger, c'est que des politiques inspirées par la nouvelle gestion publique servent d'instruments pour l'implantation d'une idéologie gestionnaire qui accroit le pouvoir managérial sans tenir compte de la réalité des organisations professionnelles (De Gaulejac, 2005). Or, pour paraphraser le titre d'un ouvrage de Chauvière (2010), «[t]rop de gestion tue le social». 
Ce qui marque particulièrement cette période, c'est certes la fin de la croissance de l'appareil d'État, la pression accrue de la demande sur des services publics - dont l'offre décline même dans certains cas - et la faiblesse de la marge de manœuvre de la plupart des organisations. Les pistes prometteuses pour favoriser l'engagement organisationnel du personnel sont connues (Fabi, Martin et Valois, 1999; Glisson, 2009; 2010).

\section{Conclusion}

"Au-delà de la reconnaissance des besoins, le problème demeure présent parce que les gestionnaires en exercice se sentent mal préparés pour offrir le type de soutien qu'ils savent nécessaire dans un contexte en constant changement qui les sollicite déjà beaucoup. »
Au-delà de la reconnaissance des besoins, le problème demeure présent parce que les gestionnaires en exercice se sentent mal préparés pour offrir le type de soutien qu'ils savent nécessaire dans un contexte en constant changement qui les sollicite déjà beaucoup. Et, contrairement au secteur privé, les organisations disposent de peu de moyens pour récompenser les conduites exemplaires.

Il y a toutefois des pratiques reconnues et attendues dans les milieux de pratique du travail social : entre autres, programme d'accueil des nouveaux employés, supervision des jeunes diplômés, pairage de ceux-ci avec des collègues expérimentés, consultation face aux problématiques complexes et groupes de pairs.

Les organisations devront aussi faire face au bouleversement majeur de leurs ressources humaines expérimentées qui partent à la retraite. Le phénomène affectera davantage les cadres. Or, très peu d'organisations disposent d'un programme de préparation de la relève et certains établissements commencent déjà à éprouver de la difficulté à recruter des personnes intéressées à assumer de telles responsabilités, étant donné que la charge de travail accrue des gestionnaires n'est pas véritablement compensée par leur niveau de rémunération. D'énormes défis en perspective à relever dans un contexte difficile. Mais n'est-ce pas là le quotidien des travailleurs sociaux? 


\section{Bibliographie}

ASSOCIATION QUÉBÉCOISE DES ÉTABLISSEMENTS DE SANTÉ ET DES SERVICES SOCIAUX (2010). Soigner l'humain pour bâtir demain, Montréal, AQESSS, 19 p.

ARCAND, Michelle, et Lorraine BRISSETTE (1998). Échec au burnout — Guide à l'intention des intervenants sociaux, Montréal, Chenelière/McGraw-Hill, $140 \mathrm{p}$.

AUBERT, Nicole, et Max PAGÈS (1989). Le stress professionnel, Paris, Éditions Klincksieck, 280 p.

BEJAN Reluca (2012). «I love my job. It's the work I hate :The erosion of social workers' employment related conditions ", [inédit], Congrès des sciences sociales et humanités 2013.

BÉLAND, Jean-Pierre (dir.) (2009). La souffrance des soignants, Québec, Presses de l’Université Laval, $87 \mathrm{p}$.

BOURQUE, Denis (2009). "La création des CSSS et certains de ses effets sur les pratiques des travailleuses sociales ", Intervention, 131, p. 161-171.

BOYER, Richard, Huguette COURTEMANCHE, Michel PRÉVILLE et Lise LACHANCE (1995). La détresse psychologique chez les infirmières et les préposé(e)s aux bénéficiaires de l'Hôpital Louis- $H$. Lafontaine, Montréal, Centre de recherche Fernand-Seguin.

BRUNET, Luc, et André SAVOIE (1999). Le climat de travail, Outremont, Les Éditions Logiques, 235 p. CHAUVIÈRE, Michel (2010). Trop de gestion tue le social, Paris, La Découverte, 232 p.

COLLERETTE, Pierre (2008). «Pour une gestion du changement disciplinée dans l'administration publique ", Télescope, Automne, Québec, École Nationale d'Administration publique, p. 33-49.

COUTURIER, Yves, et Barbara LEGAULT (2002). " Du doute identitaire à la complexité de la tâche ", Intervention, 115, p. 57-63.

DE GAULEJAC,Vincent (2005). La société malade de la gestion, Paris, Seuil, 280 p.

FABI, Bruno,Yves MARTIN et PierreVALOIS (1991). «Favoriser l'engagement organisationnel des personnes œuvrant dans des organisations en transformation ", Gestion, Vol. 24, No 3, p. 102-113.

GLISSON, Charles, et Anthony HEMMELGARN (1998). «The effects of organizational climate and interorganizational coordination on the quality and outcomes of children's services ", Child abuse and neglect, Vol. 22, No 5, p. 401-421.

GLISSON, Charles (2009). "Organizational climate and culture and performance in the human services ", dans Rino Patti (dir.), Handbook on social welfare management, Thousand Oaks, CA, Sage Publications, p. 119-141.

GLISSON, Charles (2010). "Organizational climate and service outcomes in child welfare agencies ", dans M.B.Webb, et collab. (dir.), Child welfare and child well-being: New perspectives from the National survey of child and adolescent well-being, New York, Oxford University Press, p. 378-406.

HIRIGOYEN, Marie-France (2001). Malaise dans le travail Harcèlement moral - Démêler le vrai du faux, Paris, La Découverte et Syros, 289 p.

HOPKINS, Anne H. (1983). Work and job satisfaction in the public sector, Totowa NJ, Rowman and Allanheld, $146 \mathrm{p}$.

LACHAPELLE, René, et collab. (2011). "Création des CSSS et transformation des pratiques psychosociales dans le programme Perte d'autonomie liée au vieillissement (PALV) », Intervention, 134, p. 35-45.

LAFLAMME, Roch (1994). La vie dans les organisations, Québec, Presses de l’Université Laval, 72 p. 
LARIVIÈRE, Claude (2001). Les progrès réalisés dans la collaboration CJ/CLSC en 2001, Montréal, Association des CLSC et des CHSLD du Québec, Association des Centres jeunesse du Québec. MINISTÈRE DE LA SANTÉ ET DES SERVICES SOCIAUX (2001). Rapport du comité provincial d'assurance-salaire, Québec, MSSS, $82 \mathrm{p}$.

MINISTÈRE DE LA SANTÉ ET DES SERVICES SOCIAUX (2010). Évaluation de l'implantation des réseaux locaux de services de santé et de services sociaux, Québec, Ministère de la Santé et des Services sociaux, $108 \mathrm{p}$.

ORDRE DES TRAVAILLEURS SOCIAUX ET DES THÉRAPEUTES CONJUGAUX ET FAMILIAUX DU QUÉBEC (2010). Les impacts de la création des CSSS sur la pratique en travail social, Montréal, OTSTCFQ.

PEZET,Valérie, Robert VILLATTE et Pierre LOGEAY (1993). De l'usure à l'identité professionnelle : le burn-out des travailleurs sociaux, Paris, T.S.A.É 